\title{
Notes on the International System (SI) of Units
}

\author{
Dep. of Mechanical Engineering \\ Faculty of Energy Engineering \\ Aswan University \\ Aswan, Egypt \\ salama_AbedelHady@hotmail.com
}

\begin{abstract}
According to many scientists; there are some redundancies in the SI system of units that is found in the non-correspondence between units of analogous parameters in the electric, magnetic and thermal fields. The main source of such conflicts is the missing clear definitions of electric charge and magnetic flux in literature that led also to errors in laws of electromagnetism. In the present study; we show such redundancies and conflicts. Then, we introduce innovative definitions of the electric charge and magnetic flux as electromagnetic waves of electrical or magnetic potentials which proved its success in robust definitions in electromagnetism to remove such redundancies. Such approach keeps the correspondence between the units of analogous parameters in the electric, magnetic and thermal fields. In this system; the Volt is used as a unique scale for measurement the potentials of the thermal, electric and magnetic fields and entropy of each field is used as indicator of the flowing energies in Joule/Volt.
\end{abstract}

Keywords: Entropy, Electric Charge, Magnetic Flux, Electromagnetic waves.

\section{Introduction:}

Many scientists have found the international system of units, SI system, as a historical, independent, and redundant system [1,2 and 3]. Using the SI system; the dimensional homogeneity is not satisfied in many physical equations and constants. In table 1 , we can find examples of such conflicts, which are found in literature [1 and 2], between the units of analogous quantities in the electric and magnetic fields. The ampere is a unit of the electric current, while "ampere $/ \mathrm{m}$ " is used as a unit of strength or potential of the magnetic fields. According to Maxwell's wave equation [4], the unit of the electric field strength, E, should be similar to the unit of the magnetic field strength, $\mathrm{H}$, as both are potentials of the flowing components of the same electromagnetic waves. However, both have conflicting SI units as seen in Table 1. Similarly; the conductivity is analogous to permeability while their SI units are confusing as also seen in table 1.

Table 1: Electric / Magnetic SI units (non) analogues in SI System

\begin{tabular}{|c|c|}
\hline Magnetic quantity & Electric quantity \\
\hline magneto-motive force Amp & electromotive force Volt \\
\hline magnetic field strength Amp/m & electric field strength V/m \\
\hline permeability $\mathrm{kg} \mathrm{m} / \mathrm{sec}^{2} / \mathrm{Amp}^{2}$ & conductivity $\mathrm{Sec}^{3} \mathrm{Amp}^{2} / \mathrm{kg} / \mathrm{m}^{3}$ \\
\hline magnetic flux $\mathrm{m}^{2} \mathrm{~kg} / \mathrm{sec}^{2} / \mathrm{Amp}$ & current Amp \\
\hline magnetic flux density $\mathrm{kg} / \mathrm{sec}^{2} / \mathrm{Amp}$ & current density Amp/m² \\
\hline reluctance $A \mathrm{mp}^{2} / \mathrm{m}^{2} \mathrm{~kg} / \mathrm{sec}^{2}$ & resistance $\mathrm{m}^{2} \mathrm{~kg} / \mathrm{sec}^{3} / \mathrm{Amp}^{2}$ \\
\hline
\end{tabular}

According to Ampere's law, the produced "E.M.F." or voltage at the open ends of the loop is dependent of the time rate of change of the magnetic flux encircled by the loop. According to electromagnetism References [5]; the found relation is expressed as follows:

$\frac{d \emptyset}{d t}=A * \frac{d B}{d t}=-V$

Such equation proves that the units of magnetic and electric potentials are identical. However; substituting the SI units of the terms in sides of Equation (5); we find the following units will result:

$m^{2} \frac{k g / \sec ^{2}}{A m b * S e c} \neq$ Volt

Hence; this equation is incorrect from the dimensional point of view. However; it will be seen and the end of this article how the units of this equation will be In this article; we introduce an entropy approach to the natures of the electric charge and magnetic flux that suggests new units in the field of electromagnetism and keeps the analogy between the thermal, electric and magnetic fields. This 
system proves its truth in removing the redundancies found in the SI system of units.

2 Analogy of Thermal, Electric and Magnetic Fields:The Fourier Law of thermal conduction is stated as follows [6, 7]:

$q=-k \nabla T$

Where $\mathrm{q}$ is the heat flux density in $\mathrm{W} / \mathrm{m} 2 . \mathrm{T}$ is the temperature in, as postulated, in volt and $\kappa$ is the thermal conductivity of materials in W/ $\mathrm{m} 2$ V.Similarly; Ohms Law of Electric conduction is stated as follows [8,9]:

$J=-\sigma \nabla \varphi_{e}$

Where $\mathrm{J}$ is the electric flux density in $\mathrm{W} / \mathrm{m} 2$. $\varphi$ e is the electrical potential in Volts and $\sigma$ is electrical resistance in $\mathrm{W} / \mathrm{m} 2 \mathrm{~V}$ or Ohm. The commonly used form for the relationship between the magnetic field parameters B and $\mathrm{H}$ is [9]:

$B=-\mu_{m} H$

$\mathrm{B}$ is sometimes called the magnetic flux density or the magnetic induction. The unit of an energy- flux density is measured generally in $\mathrm{W} / \mathrm{m} 2$. Hence, the unit of B should be analogous to the units of the thermal and electrical fluxes in $\mathrm{W} / \mathrm{m} 2$. $\mu \mathrm{m}$ is the magnetic permeability of a material that is analogous to the thermal and electrical conductivities. By analogy between the electric, magnetic and thermal field [4], equation (3) that describes the magnetic flux can be expressed by an equation similar to equations (1) and (2) of the form [8,9]:

$B=-\mu_{m} \nabla \varphi_{m}$

Comparing equations (3) and (4), the magnetic potential $\varphi \mathrm{m}$ can be defined in terms of the magnetic field intensity by the following relation:

$$
H=-\nabla \varphi_{m}
$$

According to equations (1), (2) and (4); the heat, electric and magnetic fluxes are denoted by $\mathrm{q}, \mathrm{J}$ and $\mathrm{B}$ and they are measured in $\mathrm{W} / \mathrm{m} 2$. Similarly, the thermal, electric and magnetic potentials are denoted by $\mathrm{T}$, $\varphi$ e and $\varphi \mathrm{m}$ and they are measured, as postulated, in Volts. Finally, the unit of conductivities $\mathrm{k}, \sigma$ and $\mu \mathrm{m}$ will result, according to the stated equations, in $\mathrm{W} / \mathrm{m} 2 \mathrm{~V}$. Such modified units of the fluxes and potentials may be considered as results of the definition of the electric charge and the magnetic flux as forms of electromagnetic waves [10].

3.An Entropy Approach to the nature of Electric Charges and Magnetic Flux: Faraday succeeded in converting electromagnetic waves, or light, unto electric current by the effect of electric field showing the electric current is electrified light or electromagnetic waves of electric potential [11]. Similarly; Tesla succeeded in transferring electric energy into space as waves [12]. Such experiments represent an evidence of the nature of electric current as flow of electromagnetic waves. Analytical and graphical proof of the conclusions from the experiments of Faraday and Tesla were verified by following by replacing the time coordinate in the Maxwell's wave equations by entropy "s." So, the Maxwell's equations were casted into an appropriate energy frame of reference as follows $[13,14]$ :

$\left(\nabla^{2}-\frac{1}{c^{2}} \frac{\partial^{2}}{s^{2}}\right) \mathrm{E}=0$,

$\left(\nabla^{2}-\frac{1}{c^{2}} \frac{\partial^{2}}{s^{2}}\right) H=0$,

In equations (8) and (9): $\mathrm{c}$ is the speed of light, $\mathrm{E}$ is the electric field and $\mathrm{H}$ is the magnetic field. Fig. 1 graphical representation of these equations where the Electric Field " $\mathrm{E}$ " is the vertical axis, the magnetic field " $\mathrm{H}$ " is the abscissa, and the entropy " $\mathrm{s}$ " is the ordinate [14]. This representation succeeds in showing the energy flow during an electromagnetic wave as the sum of the areas swept by the electric and the magnetic waves. So, the flowing electric energy per an electromagnetic wave is expressed by the first integral and the flowing magnetic energy per wave is represented by the following equation [15].

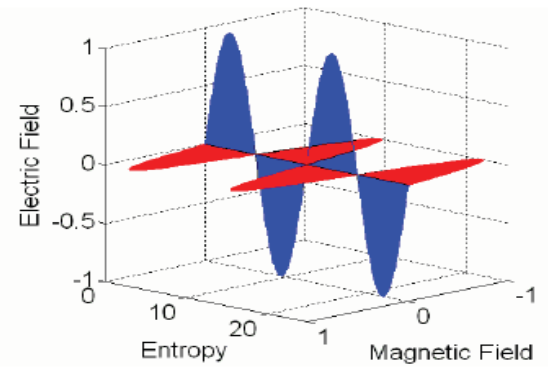

Fig. 1: Flow of electromagnetic waves in an energy frame of reference that shows the electric energy flow in an Electric field- Entropy plane and the magnetic energy flow into a Magnetic field - Entropy plane [15].

$\mathrm{Q}_{\text {total }}=\mathrm{Q}_{\mathrm{el}}+\mathrm{Q}_{\mathrm{mag}}=\int_{0}^{2 \pi}|\mathrm{E} \mathrm{dS}| \int_{0}^{2 \pi}|\mathrm{H} \mathrm{dS}|$

Joule/wave 
According to previous works; it has been found that this sum which represents the quantity of energy flow per wave is equal to Planck's constant, h Joule/wave [16]. Such results are confirmed by results of the early Planck's experiments [17]. (Accordingly, the quantity "h u" Watt represents the rate of the energy flow of pulses, or quanta of magnitude " $h$," of electromagnetic waves of frequency "v" [18].

By the introduced modification of Maxwell's equation (1) and (2), it was possible to find an analytical representation of the electric current as a special solution of such equations. Equation (3) represents a special solution of the differential Eq. (1) as an electromagnetic wave whose electric field "E" has gained an initial potential " + / $-\Delta \bar{E} "$ as follows [19].

$$
\mathrm{E}(\mathrm{r}, \mathrm{s})=\mathrm{E} \cos (\mathrm{kr}+\omega \mathrm{s}+\varphi)+/-\Delta \overline{\mathrm{E}}(11)
$$

$\mathrm{H}(\mathrm{r}, \mathrm{s})=\mathrm{H} \cos (k r+\omega s+\varphi)$

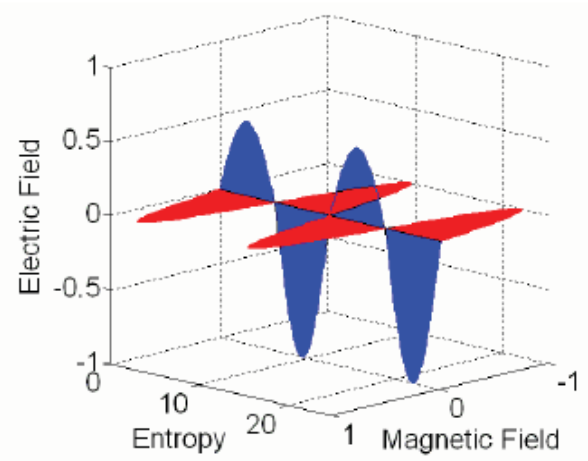

Fig. 2. Flow of electric charges as E.M. waves of non-zero electric potential [52].

Such solution of the Maxwell's equation is represented graphically for flow of negative charges in Fig. 2 A represents the electric current as a flow of negative charges in the form of electromagnetic of negative electric potential $(-\Delta \bar{E})$ in a frame of energy coordinates $E, H$, and S. Fig. 2 B shows also the electric current as flow of positive electric charges in the form of electromagnetic of positive electric potential $(+\Delta \overline{\mathrm{E}})$ in a frame of energy coordinates $\mathrm{E}, \mathrm{H}$, and $\mathrm{S}$. Both figures represents visualization of the electric current as electromagnetic or energy waves whose quantity is " $h$ " $\mathrm{J} /$ wave, whose frequency is " $\mathrm{v}$ " and whose electric potential is " + / $-\Delta \overline{\mathrm{E}}$. So, the electric pulses may have negative or positive potential, " $+/-\Delta \overline{\mathrm{E}}$ " which determine the location of the axis of its oscillations [9]. So, this figure defines the electric energy as flow of ionized quanta of energy; each of magnitude "h J/wave" and a potential of the value " $+/-\Delta \overline{\mathrm{E}}[20]$. The flow of magnetic energy is represented by the following equation as flow of electromagnetic waves of magnetic potential:

$\mathrm{E}(\mathrm{r}, \mathrm{s})=g_{1}(\omega \mathrm{s}-\mathrm{kr})$

$\mathrm{H}(\mathrm{r}, \mathrm{s})=g_{2}(\omega \mathrm{s}-\mathrm{kr})+/-\Delta \mathrm{H}$

Graphical representation of this equation is shown in Fig. ${ }^{\top}$.

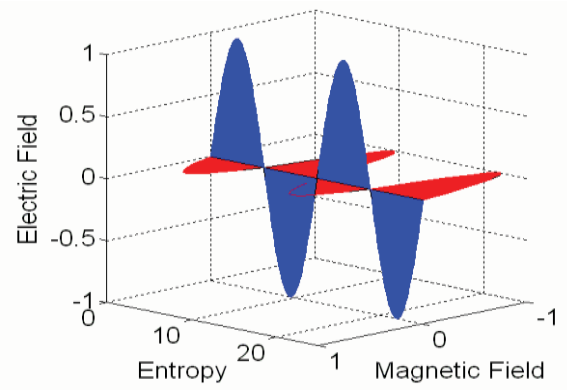

Fig.3: Graphical representation of magnetic flux [14].

A Fundamental Equation of Thermodynamics According to a previous study it has been introduced a fundamental equation of energy as follows [21]:

$$
\begin{aligned}
& d \boldsymbol{U}=\delta Q_{\text {th }}+\delta Q_{\text {elect }}+\delta \Phi_{\text {mag }}-\mathbf{p d V}+\sigma \mathrm{d} \boldsymbol{\mathrm { d }}+ \\
& \sum \boldsymbol{\mu}_{\mathrm{i}} \mathbf{d} \mathbf{n}_{\mathrm{i}}
\end{aligned}
$$

According to the second law of T.D., the flow of heat into a system generates an entropy increase of such system expressed by the relation [21]:

$Q_{\text {thermal }}=\int T d S_{\text {thermal }}$

According to the previous analysis and the derived similarities, the magnetic flux transferred to a system can be expressed also as follows [21]:

$\Phi_{\text {mag }}=\int \mathbf{B} \mathbf{d} \mathbf{S}_{\text {magnetic }}$

Similarly; the electric charge can be expressed by a similar expression as follows [22]:

$\mathbf{Q}_{\text {elect }}=\int \mathbf{E} \mathbf{d} \mathbf{S}_{\text {electric }}$

Substituting these quantities in Eq. (15); we get a fundamental equation of thermodynamics in the following form:

$d U=T d S+E d S+H d S-P d V$ 
Interchangeability of Energies:

Reviewing Faraday's experiment of magnetic induction, the electric current in the primary coil, loop 1 , is converted by induction into magnetic flux in the core; then the induced magnetic flux of the core is converted once more into an electric current in the secondary coil, loop 2, Fig. 4 [23]. Such understanding of the results showed that both fluxes, electric and magnetic, should have similar natures and that their conversion process is concluded by exchanging their potentials, electric or magnetic, firstly in the primary coil and after in the secondary coil [24].

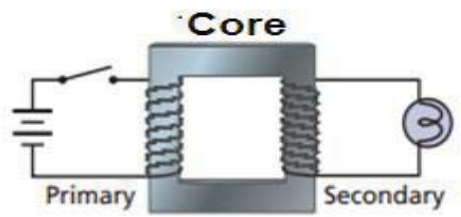

Fig. 4. Faraday's Experiment

According to these experimental results; it is possible to state that the electric current, magnetic flux and thermal radiation have the same nature as electromagnetic waves of different potentials.

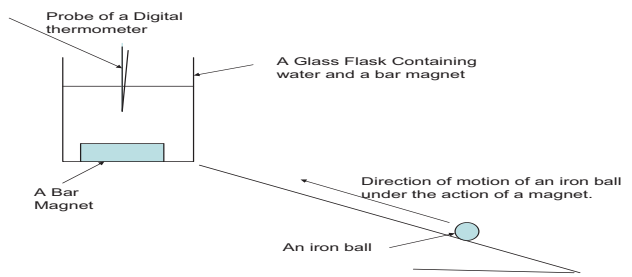

Fig. 5. Interchangeability of Magnetic and Thermal Energy .

Reviewing the results of a simple experiment, Fig. 5 [25], that used a permanent magnet immersed in a water basin to attract iron balls along an inclined smooth glass plane; the heat lost from the water was found equivalent to the done magnetic work in attraction of the balls along the shown plate [26]. Such results may be considered as a proof that magnetic work used to attract the balls is equally substituted by the heat lost from the water; or that potential of heat is replaced by magnetic potential to substitute the lost magnetic energy from the magnet. Similarly, it was possible to measure the equivalence of the heating rates, in magnetic fluids, to the subjected alternating magnetic power [27]. Reviewing also the definition of the magnetocaloric effect in literature as applied in refrigeration; it is possible to deduce the same conclusion of similarity of the natures of heat and magnetic flux [28]. Consequently, it is possible to postulate the similarity of the natures of the magnetic flux and heat and their reversible interchangeability through exchanging their potentials [29].
As a final conclusion; heat, electric and magnetic energies were concluded as forms of energies of similar and of equivalent natures and that they are reversibly interchangeable by exchanging their potentials through reversible phenomena as thermoelectric effect, magnetocaloric effect, and induction.

Accumulation of PotentialsDuring their experimental that used Aluminum-Iron thermocouples; they recorded effects of electric and magnetic field on the produced EMF by temperature differences. Fig. 6 shows the influence of application of electric field of potential 4 Volts on the generated EMF and Fig. 7 shows the effect of applying magnetic field on the generated EMF [31]. Such results sustained the definition of the electric current and magnetic flux as electromagnetic waves that possess electric potential.

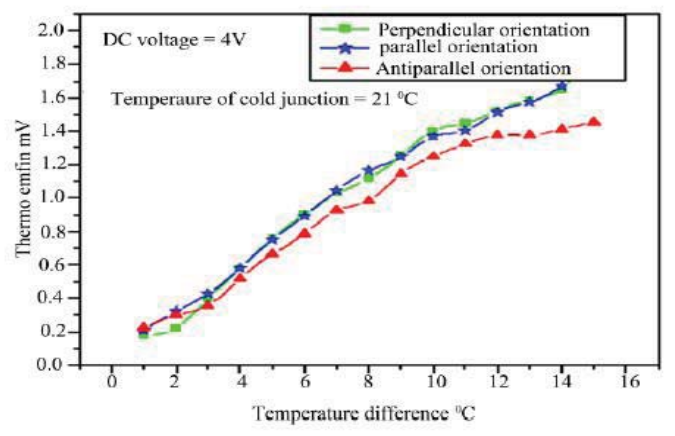

Fig. 6. : Influence of the Electric Field on the produced EMF in Thermocouple measurements [30].

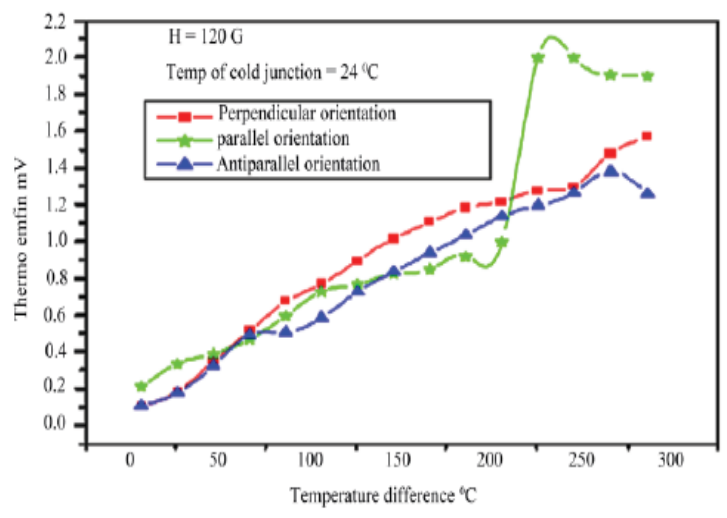

Fig. 7: Influence of the Magnetic Field on the produced EMF in Thermocouple measurements [30].

Such scales assure the use of the same unit to measure the potentials of the thermal, electric and magnetic energies as can be concluded from the following equation that can be found by rearranging the terms of Eq. (19) as follows [31]: 
$d U=(T+E+H) d S-\delta W$

According to the thermocouple experiments; it is possible to introduce new forms of the Seebeck coefficient which involves the influence of the electric or magnetic field in addition to the influence of the temperature difference that can be defined by the following equations in case of applying electric field of strength $E$ or magnetic field of strength $\mathrm{H}[32]$ :

$\Delta V=\propto_{e A B}\left(T_{h}-T_{l}\right)+E$

$\Delta V=\propto_{m A B}\left(T_{h}-T_{l}\right)+H$

Modification of the SI system of units:

Introducing the same unit for the thermal and electric potentials; It is possible to find the analogy between different units that are used in electromagnetism according to the following Table.

Table 2.: Electric / Magnetic innovative analogues units

\begin{tabular}{|c|l|}
\hline $\begin{array}{l}\text { Magnetic } \\
\text { quantity }\end{array}$ & $\begin{array}{l}\text { Electric } \\
\text { quantity }\end{array}$ \\
\hline $\begin{array}{l}\text { Magneto- } \\
\text { motive } \\
\text { force } \\
\text { Volt }\end{array}$ & $\begin{array}{l}\text { Electromotive } \\
\text { force } \\
\text { Volt }\end{array}$ \\
\hline $\begin{array}{l}\text { Magnetic } \\
\text { field } \\
\text { strength } \\
\text { Volt/m }\end{array}$ & Electric field \\
strength \\
Volt/m
\end{tabular}

According to the definition of electric charge and magnetic flux plainly as electromagnetic waves of electric or magnetic flux; we delete actually the ambiguous units as Coulomb, Gauss, and Ampere. However; we can find that ammeter measures actually the rate of flow of entropy not the rate of flow of electrons as found in literature.
A Proof of Faraday's Law of Induction and Modification of Ampere's Law. As the rate of flow of Energy is the same through the iron-frame. However; if the primary coil is actuated by electric potential $E_{p}$, the rate of flow of entropy through the iron frame will be defined according to Eq. (17) by the following equation [21]:

$\dot{W}=E_{p} \dot{S}$

When using $\mathrm{n}$ turns in the secondary coil; the share of each turn from the entropy flow will be $\frac{1}{n}$ of the total entropy; i.e. $\frac{\dot{s}}{n}$ According to the principles of conservation of energy; the generated power from the secondary coli should be equal to the output energy. Hence; we may write:

$\dot{W}=E_{p} \dot{S}=E_{s} * \frac{\dot{s}}{n}$

Hence; the potential of the secondary coil will be:

$E_{s}=n E_{p}$

This means that each turn will produce the same potential applied to the primary coil of single turn. Such relation is found in literature without a similar plausible explanation as has been derived by recognizing the natures of electric and magnetic fields as electromagnetic waves of electric or magnetic potentials.

To correct the dimensional corruptions in Ampere's law; the proportionality coefficient between the E.M.F. or voltage at the open ends of the loop and the time rate of change of the magnetic flux encircled by the loop will be the reluctance of the loop ; i.e.

$A * R_{m} * \frac{d B}{d t}=-V$

By using the new system of units and inserting the reluctance on the L.H.S. of the equation. Ampere's law can be rewritten in adjustable dimensional form where the coefficient as follows:

$m^{2} * \frac{\text { Volt }}{W} * \frac{W}{m^{2}} \equiv$ Volt

\section{References:}

[1] G. M. Trunov, "Correctness of the International System of Units in the Area of Electromagnetism," Journal of Measurement Techniques, Vol. 26, No. 1, 1983, pp. 9-10.

[2] G. D. Yarnold, "Notes on Electric and Magnetic Dimensions," Proceedings of the Physical Society, iopscience, iop.org, 1942, pp. 189-193 
[3] Salama Abdelhady, " Comments concerning Measurements and Equations in Electromagnetism." "J. Electromagnetic Analysis \& Applications", November, 2010, Vol. 2: pp.217-219.

[4].J. Jewett and R. A. Serway, "Physics for Scientists and Engineers with Modern Physics," 7th Edition, Thomson, Brooks/Cole, 2008.

[5] M. Fujimtu, Physics of Classical Electromagnetism, Springer, 2007

[6] R. Heikes, W. Roland, and J. Ure, Thermoelectricity: Science and Engineering. Interscience Publishers Inc., 1961, New York.

[7] S. B. Riffat,and Ma, X. Thermoelectrics: A Review of Present and Potential Applications. 2003, Applied Thermal Engineering

[8] Bruce Sherwood. P. J. Scanlon, R. N. Henriksen, and J. R. Allen, "Approaches to electromagnetic induction," Am. J. Phys. 377, 698-708 1969.

[9] Buist, R.J. (1995) The Extrinsic Thomson Effect. Proceedings of the 14th International Conference on Thermoelectrics,St. Petersburg, 27-30 June 1995, 27-30.

[10] Tschoegl, N., Fundamentals of Equilibrium and Steady-state Thermodynamics; Elsevier Science, Ltd: Amsterdam, The Netherlands, 2000

[11] Ryan, D.T. (2006) Toward a Cognitive-Historical Understanding of Michael Faraday's Research: Editor's Introduction.Perspectives on Science, 14, 1-6.

[12] Salama Abdelhdy, "A Fundamental Equation of Thermodynamics that Embraces Electrical and Magnetic Potentials' "J. Electromagnetic Analysis \& Applications", March, 2010, Vol. 2: pp. 162-168.

[13] E. G. Thomas and A. J. Meadows, Maxwell's Equations and their Applications, Adam Hilger Ltd.,Bristol and Boston (1985).

[14] Lebowitz J.L., Macroscopic Laws and Microscopic Dynamics, Time's Arrow and Boltzmann's Entropy. Physica A, 1993( 194), 1-97

[15] Salama Abdelhady, Thermodynamic Analysis of Electric Charges and Magnetic Flux, Cairo 11th International Conference on Energy and Environment, Ghurgada, Egypt, March, 2009, 175-185

[16] Stevens, C.F. (1965) The Six Core Theories of Modern Physics. MIT Press, Cambridge.

[17]Feynman, R.P., Leighton, R.B., and Sands, M. Lectures on Physics, Volume 1. Addison-Wesley, Reading MA (1963).

[18] Haaiday, D., Resnick, R. and Walker, J., Fundamentals of Physics. 7th Edition, John Wiley \& Sons, New York, 2004
[19] S. Abdelhady, "An Entropy Approach to Optical Pumping," Proceedings of the 15th International Conference on Aerospace Sciences \& Aviation Technology, ASAT-15, Cairo, May 2012

[20] S. Abdelhady, “An Entropy Approach to Wireless Power Transmission by Magnetic Resonance," Journal of Applied Physics, 06/2013; 5(5):pp. 29 - 35. DOI:10.559/apr.v5n5p29

[22] Salama Abdelhady, "An Advanced Review of thermodynamics of Electromagnetism," International Journal of Research studies in Science, Engineering and Technology, Vol. 3, No. 6, 10, 2015.

[23] Harman T.C., Honig J.M., Thermoelectric and Thermomagnetic Effects and Applications; McGraw-Hill Book Company, 1967

[24] Salama Abdelhady, "Innovative Understanding of the Duality confusion, the Photovoltaic and Magnetocaloric Effects." Ain Shams Engineering Journal, Engineering Physics and Mathematics, Vol. 8, March 2017.

[25] Salama Abdelhady, Review of Thermodynamics of Systems that Embrace Transfer of Electric and Magnetic Energies, Journal of Physical Science and Application, doi: 10.17265/2159-5348/2018.01.001, 8, 1, 1, (2018)

[26] Salama Abdelhady, Thermodynamics: Fundamentals and its Application in Science, Auris Reference; 1st edition,1,(2017), ISBN-10: 1788020626 An International Text Book In Science.

[27] Abdelhady S., An entropy approach to wireless power transmission by magnetic resonance. Appl. Phys (2013).

[28] Phan MH, Yu SC. Review of the magnetocaloric effect in manganite materials. J. Magn Magn Mater( 2007);308(2):325-40

[29] Peksoy O, Rowe A. Demagnetizing effects in active magnetic regenerators. J Magn Mat 2005;288:424.

[30] V. Kumar, J. Singh and S. S. Verma, "Performance Comparison of Some Common Thermocouples for Waste Heat Utilization," Asian Journal of Chemistry, Vol. 21, No. 10, 2009, pp. 62-65

[31] Abdelhady S. An approach to a universal system of units. J Electromagn Anal. Appl (2010);2:549.

[32] Wang B, Kucukgok BD, Leach Melton J, Udwary K, Evans K, Lu N, et al., Thermoelectric properties of undoped and si-doped bulk GaN. In: MRS proceedings, vol. 1558, (2013) 